11,04

\title{
Калибровочная теория стекольного перехода в трехмерной XY-модели с вмороженным беспорядком
}

\author{
(C) М.Г. Васин ${ }^{1,2}$, В.М. Винокур ${ }^{3}$ \\ ${ }^{1}$ Удмуртский ФИЦ УрО РАН, \\ Ижевск, Россия, \\ ${ }^{2}$ Институт фризики высоких давлений им. Л.Ф. Верещагина РАН, \\ Москва, Россия \\ ${ }^{3}$ Аргонская национальная лаборатория, \\ Чикаго, США \\ E-mail: dr_vasin@mail.ru
}

Рассмотрена калибровочная теория фазовых переходов в трехмерной ХҮ-модели с закаленным беспорядком. Показано, что топологический фазовый переход, происходящий в вихревой подсистеме данной модели, представляет собой переход в состояние спинового стекла.

Работа выполнена при поддержке гранта РФФИ 18-02-00643.

DOI: 10.21883/FTT.2018.06.45998.26M

\section{1. Введение}

Природа перехода жидкость-стекло до сих пор до конца не понята и вызывает дискуссии среди специалистов из различных областей физики. Стеклообразование является общим свойством различных по своей природе физических систем: жидкостей с сильными ковалентными связями, таких как обычные силикатные стекла; жидкостей с водородными связями, таких как глицерин; спиртов, со слабыми ван-дер-Ваальсовскими связями; ионных жидкостей; спиновых и вихревых систем, включая сверхпроводники второго рода; и даже биологических систем. Однако до сих пор не существует теории, позволяющей даже на качественном уровне одновременно описать наиболее характерные свойства стекольного перехода. По этой причине задача разработки такой теории до сих пор остается актуальнейшей задачей физики конденсированного состояния.

Тем не менее, появилась надежда на решение данной проблемы, связанная с использованием квантовополевых и функциональных методов теоретического описания перехода жидкость-стекло. Основы данного теоретического подхода были заложены в конце прошлого века. Тогда были сформулированы методы описания стекольных систем с помощью калибровочной теории поля: поскольку фрустрированная система инвариантна относительно локальных поворотов фрустрированных областей структуры $[1,2]$, описывающая ее теория также должна быть инвариантной относительно данных локальных преобразовний. При описании таких систем в непрерывной модели возникают калибровочные поля. Впервые на локальную (калибровочную) инвариантность фрустрированных систем обратил внимание Тулуз [3], после чего калибровочная теория была развита для описания спин-стекольных систем в $[1,4,5-7]$.
Следует заметить, что примерно в то же время появились работы Березинского, Костерлица и Таулеса (БКТ) [8-10], которые принесли в физику конденсированного состояния новую плодотворную концепцию топологических фазовых переходов, происходящих в системе вихрей (топологических солитонов). Среди свойств перехода БКТ выделяется особенность его критического поведения. При приближении сверху к критической температуре, $T=T_{\mathrm{BKT}}$, корреляционный радиус очень быстро расходится, $\xi \sim \exp \left[\sqrt{E_{0} /\left(T-T_{\mathrm{BKT}}\right)}\right]$, намного быстрее любого степенного закона, $\xi \sim\left|T-T_{c}\right|^{-v} \quad\left(T_{c}-\right.$ критическая температура перехода, $v>0-$ критический индекс), определяющего корреляционный радиус для стандартного непрерывного фазового перехода $[11,12]$. Это поразительно напоминает критическую расходимость, наблюдаемую при стекольном переходе, когда характерное время корреляции подчиняется известному закону Фогеля-Фулчера-Таммана (ФФТ), $\tau \sim \exp \left[E_{0}^{\prime} /\left(T-T_{\mathrm{G}}\right)\right]\left(T_{\mathrm{G}}\right.$ - температура стеклования $)$, и приводит к предположению о глубокой взаимосвязи между двумя этими явлениями [13].

Для исследования этого вопроса в представленной работе рассмотрена калибровочная теория топологического фазового перехода в ХҮ-модели с закаленным беспорядком. Стеклообразование в трехмерной ХY-модели было предметом многочисленных исследований, основанных как на методах квантовой теории поля $[5,6,4,7]$, так и на вычислительных методах, см., например, [14-17]. В частности, было промоделировано медленное охлаждение трехмерной ХҮ-модели и продемонстрировано, что система с первоначально случайно распределенными спинами замораживается в состоянии вихревого стекла [18]. Тем не менее, несмотря на значительные усилия, сам процесс стеклования и его связь с топологическими возбуждениями в XY-системах все еще остается малоизученным. 


\section{2. Формулировка модели}

В настоящей работе мы рассмотрим трехмерную ХY-модель, представляющую собой трехмерную сетку, в узлах которой, r, находятся двухкомпонентные классические векторы единичной длины, которые могут вращаться в плоскости XY. Гамильтониан системы представляется в виде

$$
H=-\frac{1}{2} \sum_{\left\langle\mathbf{r} \neq \mathbf{r}^{\prime}\right\rangle}^{N} \varepsilon_{\mathbf{r}-\mathbf{r}^{\prime}} \mathbf{S}_{\mathbf{r}} \cdot \mathbf{S}_{\mathbf{r}^{\prime}}
$$

где $\varepsilon_{\mathbf{r}-\mathbf{r}^{\prime}}-$ положительный коэффициент взаимодействия между ближайшими соседями, $\varepsilon=$ const $>0, N-$ общее число узлов, а скобки $\left\langle\mathbf{r} \neq \mathbf{r}^{\prime}\right\rangle$ означают суммирование по ближайшим соседям вокруг r. Присутствие закаленного беспорядка моделируется тем, что коэффициент взаимодействия случайным образом с некоторой вероятностью может принимать отрицательное значение, $\varepsilon<0$.

Общие свойства этой модели в отсутствие вмороженного беспорядка хорошо изучены и существенно зависят от размерности системы. В системе с размерностью $d \leqslant 2$ тепловые флуктуации всегда разрушают дальний порядок, как доказали Мермин, Вагнер [19] и Хоенберг [20] из неравенств Боголюбова. Тем не менее, локально спины упорядочены. Действительно, хотя при высоких температурах система полностью разупорядочена, при ее охлаждении ниже некоторой температуры $T_{c}$ возникает локальное ферромагнитное упорядочение. Однако это не приводит к возникновению дальней трасляционной симметрии, поскольку система содержит разрушающие дальний порядок подвижные топологические возбуждения. Далее мы будем называть эти возбуждения „вихри“ по аналогии с вихрями в сверхтекучем гелии, которые, кроме того, являются братьямиблизнецами дисклинаций в теории упругости. Таким образом в системе формируется кулоновский газ вихрей, в котором при дальнейшем уменьшении температуры до $T=T_{\mathrm{BKT}} \equiv T_{g}<T_{c}$ происходит топологический фазовый переход БКТ. В качестве параметра, характеризующего данный переход, выступает радиус корреляции вихрей, который критически расходится при $T \rightarrow T_{g}$. При этом, как уже было отмечено, в низкотемпературной фазе дальний порядок отсутствует, т. е. $\langle\mathbf{S}\rangle=0$. При $d>2$ физическая картина упрощается, поскольку при критической температуре $T=T_{c}$ система претерпевает фазовый переход второго рода из высокотемпературной разупорядоченной так называемой симметричной фба$3 b l$, в которой спины свободно вращаются под действием тепловых флуктуаций, в низкотемпературную упорядоченную базу.

Ниже мы покажем, что в присутствии вмороженного беспорядка поведение трехмерной системы меняется. Для описания такой системы мы воспользуемся подходом, объединяющим пертурбативные методы, применяемые к фазовому переходу второго рода в спиновой системе, и непертурбативные методы. Мы построим нашу теорию на основе теории Вейсса в длинноволновом приближении. В этом случае плотность гамильтониана чистой ХY-модели

$$
\mathscr{H}_{0}=\frac{1}{2}|\nabla \Psi|^{2}-\frac{1}{2} m^{2}|\Psi|^{2}+\frac{b}{4}|\Psi|^{4},
$$

где $m^{2}=\alpha\left(T_{c}-T\right), \alpha$ и $b-$ положительные константы, векторное поле $\Psi_{\mathbf{r}}=\left\langle\mathbf{S}_{\mathbf{r}}\right\rangle_{\delta V}$, играющее роль поля Хиггса, есть результат усреднения спинов в некотором конечном объеме $\delta V$ вблизи точки r. Далее, для упрощения записи, мы будем опускать указатель координаты r, подразумевая, что $\Psi$ - значение поля в некоторой точке пространства. Теперь гамильтониан $H=\int \mathscr{H}_{0} \mathrm{~d}^{d} r$, в котором интегрирование выполняется по объему системы, соответствует пертурбативной теории фазовых переходов второго рода Гинзбурга-Ландау.

Важным фактом является то, что системы, описываемые формулой (2), в низкотемпературном состоянии могут содержать топологически нетривиальные вихревые возбуждения. Эти возбуждения, которые согласно теории БКТ управляют физикой двумерной системы, не учитываются в пертурбативных подходах, поскольку $\nabla \Psi \sim \psi r^{-1}$, и при наличии вихря первый член дает бесконечный вклад в энергию, $\int|\nabla \Psi|^{2} \mathrm{~d}^{d} r \sim r^{d-2} \rightarrow \infty$ при $r \rightarrow \infty$. Чтобы исправить это, вводится ковариантная производная вместо обычной, $\nabla \rightarrow D=\nabla-i g \mathbf{A}$, где A - компенсирующее поле, а $g$ - константа связи [21]

$$
\mathscr{H}_{0}=\frac{1}{2}|D \Psi|^{2}-\frac{1}{2} m^{2}|\Psi|^{2}+\frac{b}{4}|\Psi|^{4} .
$$

Далее мы представим вектора $\Psi$ в виде комплексных величин: $\Psi=\psi e^{i \Phi}$, где $\psi-$ модуль вектора $\Psi$, а $\Phi-$ фаза, соответствующая углу поворота этого вектора относительно некоторого выделенного направления. Равновесное значение $|\Psi|^{2}$ равно нулю при $T>T_{c}$ и $|\Psi|^{2}=\psi^{2}=\alpha\left(T_{c}-T\right) / b$ при $T<T_{c}$. Рассмотрим систему в низкотемпературной области. Флуктуации локального модуля намагниченности в этой области температур малы, $\nabla \psi \approx 0$, кроме того, $\nabla \mathbf{A}=0$, поэтому гамильтониан системы (3) в низкотемпературной области запишется в следующем виде

$$
\mathscr{H}_{0}=\frac{1}{2} \psi^{2}\left(|\nabla \Phi|^{2}-g^{2} \mathbf{A}^{2}\right)
$$

Теперь положим, что система содержит вихри. При обходе по контуру вокруг вихря фаза $\Phi$ меняется (увеличивается или уменьшается) на $2 \pi$. Тогда поле $\Phi$ может быть представлено в виде суммы плавно флуктуирующей, $\Phi_{S}$, и вихревой, т.е. закручивающейся на $2 \pi, \Phi_{V}$, частей, $\Phi=\Phi_{S}+\Phi_{V}$. Используя преобразование Хаббарда-Стратановича $[22,23]$ и пренебрегая полными производными можно переписать предыдущее выражение в следующем виде

$$
\mathscr{H}_{0}=\frac{1}{2} \mathbf{B}^{2}+i \Phi_{S} \nabla(\psi \mathbf{B})+i \psi \mathbf{B} \nabla \Phi_{V}-\frac{1}{2} g^{2} \psi^{2} \mathbf{A}^{2}
$$


Производя усреднение в статистической сумме по $\Phi_{S}$, получаем $\nabla(\psi \mathbf{B})=0$. Чтобы последнее равенство всегда выполнялось надо ввести новое поле $\Lambda$, такое, что $\mathbf{B}=\nabla \times \Lambda$, тогда

$$
\begin{aligned}
\mathscr{H}_{0}= & \frac{1}{2} \mathbf{B}^{2}+i \nabla\left(\psi\left(\Lambda \times \nabla \Phi_{V}\right)\right)+i \Lambda\left(\nabla \psi \times \nabla \Phi_{V}\right) \\
& +i \psi \Lambda\left(\nabla \times \nabla \Phi_{V}\right)-\frac{1}{2} g^{2} \psi^{2} \mathbf{A}^{2} .
\end{aligned}
$$

Второе слагаемое здесь будет равно нулю, если $\Lambda \propto \nabla \Phi_{V}=g \mathbf{A}$, поэтому выберем $\Lambda=\mathbf{A}$. Третье слагаемое пренебрежимо мало из-за множителя $\nabla \psi$. Четвертое слагаемое не равно нулю, когда поле $\Phi_{V}$ сингулярно. Соответствующий вихрю источник калибровочного поля $J=J_{z}=2^{-1} \psi \nabla \times \nabla \Phi_{V}$ (ось $z$ нормальна плоскости XY) принимает дискретные значения, так как при обходе вокруг сингулярности происходит приращение фазы $2^{-1} \oint \nabla \Phi_{V} d \mathbf{l}=J_{z}=\pi l(l \in \mathbb{Z})$. Заметим, что неоднородное калибровочное поле А появляется только при наличии его источников - вихрей. Если система содержит $N$ вихрей, $\mathbf{J}_{n},(n=1 \ldots N)$, тогда плотность гамильтониана принимает вид

$$
\mathscr{H}_{N}=\mathscr{H}_{0}+i \sum_{n=1}^{N} \mathbf{J}_{n} \cdot \mathbf{A}
$$

где

$$
\mathscr{H}_{0}=\frac{1}{2}(\nabla \times \mathbf{A})^{2}-\frac{1}{2} M_{0} \mathbf{A}^{2}
$$

- гамильтониан свободного калибровочного поля, $M_{0}^{2}=g^{2} \alpha\left(T_{c}-T\right) / b$ - квадрат массы калибровочного поля, возникающий из-за механизма Андерсона-Хиггса (масса Хиггса). Используя тождество $\nabla \times(\nabla \times \mathbf{A}) \equiv$ $\equiv \nabla(\nabla \mathbf{A})-\nabla^{2} \mathbf{A}$, и свойства калибровочной симметрии, $\mathbf{A} \rightarrow \mathbf{A}+\nabla f$, получаем

$$
\mathscr{H}_{0}=\frac{1}{2} \mathbf{A} \nabla^{2} \mathbf{A}-\frac{1}{2} M_{0} \mathbf{A}^{2}
$$

В импульсном представлении гамильтониан системы, содержащей $N$ вихрей, имеет следующий вид:

$$
\mathscr{H}_{N}=-\frac{1}{2} \mathbf{A}(\mathbf{p})\left(\mathbf{p}^{2}+M_{0}^{2}\right) \mathbf{A}(-\mathbf{p})+i \sum_{n=1}^{N} \mathbf{J}_{n} e^{-i \mathbf{p r}_{n}} \cdot \mathbf{A}(\mathbf{p}),
$$

а корреляционная функция свободного калибровочного поля записывается как

$$
\langle\mathbf{A}(\mathbf{p}) \mathbf{A}(-\mathbf{p})\rangle_{A}=\frac{-\beta^{-1}}{\mathbf{p}^{2}+M_{0}^{2}},
$$

где $\langle\ldots\rangle_{A}$ означает статистическое усреднение по всем возможным конфигурациям поля $\mathbf{A}$, и $\beta=1 / k_{b} T$ $\left(k_{b}\right.$ - постоянная Больцмана). Характерный корреляционный радиус пропорционален обратной массе Хиггса, $\xi \sim M_{0}^{-1}$. При $M_{0} \rightarrow 0$ вихри ведут себя как токи в магнитостатике. Гамильтониан системы, содержащий два одинаково заряженных вихря с координатами $\mathbf{r}_{1}$ and $\mathbf{r}_{2}$, это $\mathscr{H}_{2}=\mathscr{H}_{0}+i \mathbf{A} \mathbf{J}_{1}-i \mathbf{J}_{2} \mathbf{A}$. Интегрируя по полю $\mathbf{A}$, приходим к закону Био-Савара:

$$
H_{2}=J^{2} \int \frac{d^{3} \mathbf{p}}{(2 \pi)^{3}} \frac{e^{i \mathbf{p}\left(\mathbf{r}_{1}-\mathbf{r}_{2}\right)}}{\mathbf{p}^{2}} .
$$

Таким образом, гамильтониан рассматриваемой системы аналогичен гамильтониану системы электрических токов в магнитостатике.

\section{3. Усреднение по статансамблю}

При $T \lesssim T_{c}$ нарушается локальная симметрия системы, т.е. происходит локальное упорядочение спинов. Однако вмороженный беспорядок приводит к возникновению в спиновой структуре вихрей, разрушающих порядок на больших масштабах. В этой области температур рассматрим вихревую систему как газ частиц, в которой вихри подвижны и могут экранировать друг друга, что приводит к перенормировке массы Хиггса. Для ее вычисления проведем усреднение по большому каноническому ансамблю „частиц“, наделенных двумя возможными безразмерными зарядами: $\sqrt{\beta a^{d-2}} J_{n}= \pm 1$ ( $a$ - радиус ядра вихря). Тогда статистическая сумма системы имеет следующий вид:

$$
\begin{aligned}
& Z=\left\langle e^{-\beta H}\right\rangle=\left\langle\left\langle e^{-\beta H}\right\rangle_{A}\right\rangle_{J}=\left\langle\int \mathscr{D} \mathbf{A} e^{-\beta \int \mathrm{d}^{3} \mathbf{r} \mathscr{H}}\right\rangle_{J} \\
& =\sum_{N=1}^{\infty} \frac{\lambda^{N}}{N !} \sum_{\left\{J_{n}\right\}} \int \mathscr{D} \mathbf{A} \int \prod_{n=1}^{N} \mathrm{~d}^{3} \mathbf{r}_{n} e^{-\beta \int \mathrm{d}^{3} \mathbf{r}\left[\mathscr{H}_{0}-i \delta^{(2)}\left(\mathbf{r}-\mathbf{r}_{n}\right) \mathbf{J}_{n}(\mathbf{r}) \mathbf{A}(\mathbf{r})\right]},
\end{aligned}
$$

где $\langle\ldots\rangle_{J}$ означает статистическое усреднение по большому каноническому ансамблю вихрей, $\int \mathscr{D A}-$ функциональное интегрирование по полю $\mathbf{A},\left\{\mathbf{J}_{n}\right\}-$ набор всех вихревых конфигураций $\mathbf{J}_{n}= \pm J, \lambda=\lambda_{0} e^{-\beta E_{c}}-$ параметр, физический смысл которого - плотность вихрей, $\lambda_{0}$ - размерный множитель плотности вихрей, $E_{c}$ - энергия вихревого кора. Заметим, что согласно топологическим законам вихрь в $3 \mathrm{D} \mathrm{SO}(2)$ системе является линейным. Поэтому, согласно принципу наименьшего действия, часть действия, содержащая источники калибровочного поля, будет иметь следущий вид:

$$
i \beta \int d^{3} \mathbf{r} \delta^{(2)}\left(\mathbf{r}-\mathbf{r}_{n}\right) \mathbf{J}_{n}(\mathbf{r}) \cdot \mathbf{A}(\mathbf{r}) \approx i \beta J_{n} a\left|\mathbf{A}\left(\mathbf{r}_{n}\right)\right|,
$$

где $a-$ радиус вихревого кора, $J_{n}-$ топологический источник. Тогда

$$
\begin{aligned}
& Z=\sum_{N=1}^{\infty} \frac{\lambda^{N}}{N !} \\
& \times \sum_{\sqrt{\beta a} J_{n}= \pm 1} \int \mathscr{D} \mathbf{A} e^{-\beta \int d^{3} \mathbf{r} \mathscr{H}_{0}} \int \prod_{n=1}^{N} \mathrm{~d} \mathbf{r}_{n} \exp \left[i \beta a J_{n}\left|\mathbf{A}\left(\mathbf{r}_{n}\right)\right|\right] .
\end{aligned}
$$


Усредняя по безразмерной величине $\sqrt{\beta a} J_{n}= \pm 1$ получаем

$$
\begin{aligned}
& Z=\sum_{N=1}^{\infty} \frac{\lambda^{N}}{N !} \int \mathscr{D} \mathbf{A} e^{-\beta \int d^{3} \mathbf{r} \mathscr{H}_{0}} \int \prod_{n=1}^{N} d^{3} \mathbf{r}_{n} 2 \cos \left[\sqrt{\beta a}\left|\mathbf{A}\left(\mathbf{r}_{n}\right)\right|\right] \\
& =\int \mathscr{D} \mathbf{A} e^{-\beta \int d^{3} \mathbf{r} \mathscr{H}_{0}} \sum_{N=1}^{\infty} \frac{1}{N !}\left(2 \lambda \int d^{3} \mathbf{r} \cos [\sqrt{\beta a}|\mathbf{A}(\mathbf{r})|]\right)^{N} \\
& =\int \mathscr{D} \mathbf{A} \exp \left(-\beta \int d^{3} \mathbf{r}\left(\mathscr{H}_{0}-2 \lambda \beta^{-1} \cos [\sqrt{\beta a}|\mathbf{A}(\mathbf{r})|]\right)\right) .
\end{aligned}
$$

Заметим, что усреднение выполняется по всем величинам точечных вихрей и их возможным положениям. Это кажется естественным в двумерном случае. В трехмерном же случае возможны только линейные вихри. Тогда, на первый взгляд, процедура суммирования по всем конфигурациям точечных вихрей представляется не корректной. Однако, хотя точечные вихри и будут учитываться при суммировании по всем возможным спиновым конфигурациям, высокая энергия таких конфигураций приведет к тому, что их вклад будет пренебрежимо мал. Другими словами, при усреднении по всем конфигурациям 3D-системы с надлежащим весом процедура усреднения автоматически „выбирает“ только линейные конфигурации вихрей. Таким образом, приведенное выше усреднение учитывает все конфигурации линейных вихрей, включая все возможные конфигурации контуров.

В результате усреднения гамильтониан системы принимает следующий вид:

$$
\mathscr{H}=\frac{1}{2} \mathbf{A} \nabla^{2} \mathbf{A}-\frac{1}{2} M_{0}^{2} \mathbf{A}^{2}-2 \lambda \beta^{-1} \cos (\sqrt{\beta a}|\mathbf{A}|)
$$

и является ни чем иным, как гамильтоновой плотностью теории синус-Гордона [24].

Хорошо известно, что в чистых (без вмороженного беспорядка) трехмерных системах с вырожденной непрерывной симметрией низкотемпературная фаза практически не содержит вихрей. В трехмерной системе энергия для создания линейного вихря так же логарифмически расходится, как и энергия точечного вихря в двумерной системе, однако тепловые флуктуации в трехмерной системе слабее и не могут разрушить возникающий дальний порядок. Таким образом, в 3D-системе долгоживущие вихри макроскопических размеров могут существовать только при наличии дополнительного возмущения, вызванного вмороженным беспорядком, фрустрирующим систему. Согласно работам Дзялошинского с соавторами $[5,6]$, устойчивые вихри соответствуют линиям фрустрации, введенным Тулузом и Виллаином $[3,25]$. Эти линии фрустрации либо индуцируются вмороженным беспорядком [26], либо являются результатом одновременного действия геометрических ограничений на систему и конкуренции между спиновыми взаимодействиями, приводящими к вырождению основного состояния [27]. Кроме того, известно, что вмороженный беспорядок уменьшает эффективную размерность. Так, в случае, когда этот беспорядок представляет собой „белый шум“, эффективная размерность $d$-мерной неупорядоченной системы понижается до $d-2$ [28]. В результате при некоторой концентрации фрустрирующих систему элементов можно ожидать, что фрустрированная трехмерная система будет вести себя как система с более низкой размерностью. Это означает, что увеличение степени фрустрации может привести к образованию топологически устойчивых вихревых возбуждений, которые существовали бы в низкотемпературной фазе аналогично вихрям в 2D-системе. В этом случае в качестве параметра, характеризующего степень фрустрации, можно рассматривать плотность вихрей, $\lambda$.

\section{4. Анализ критического поведения системы}

4.1. Влияние вмороженного беспорядка на критическое поведение вблизи $T_{c}$. Рассмотрим теперь критическое поведение ХY-модели с вмороженным беспорядком при $T \lesssim T_{c}$. Присутствие в системе индуцированных фрустрацией вихрей приводит к необходимости учета последнего нелинейного слагаемого в (17). Можно разложить косинус в степенной ряд Тейлора. Тогда оказывается, что трехмерная система значительно проще двумерной, поскольку в этом случае релевантными оказываются не все члены степенного ряда, как в 2D-системе, а только первые два слагаемых, со степенями $A$, меньшими $q=6(q<2 d /(d-2))$ [21]. Кроме того, оказывается, что и оставшаяся эффективная нелинейность экспоненциально мала [29], $\beta \lambda \sim e^{-\beta E_{c}} \ll 1$, и удовлетворяет условию дебаевского приближения, то есть объем Дебая, $V_{D}$, содержит достаточно много частиц, что позволяет пренебречь флуктуациями суммы их полей. Действительно, поскольку плотность частиц $\lambda \propto e^{-\beta E_{c}}$, то из (17) число частиц в дебаевском объеме $V_{D} \sim\left(M_{0}^{2}+a \lambda\right)^{-3 / 2} \propto e^{3 \beta E_{c} / 2}$ экспоненциально расходится: $\lambda V_{D} \sim e^{\beta E_{c} / 2} \gg 1$. Это означает, что при $d>2$ ряд возмущений теории синус-Гордона не содержит инфракрасных расходимостей [30]. Следовательно, свободная плотность энергии системы принимает вид

$$
\mathscr{H}=\frac{1}{4} \mathbf{A} \nabla^{2} \mathbf{A}-\frac{M_{\text {eff }}^{2}}{4} \mathbf{A}^{2},
$$

где эффективное значение квадрата массы векторного поля равно перенормированной массе Хиггса, $M_{0}^{2} \rightarrow M_{\text {eff }}^{2}=g^{2} \alpha\left(T_{c}-T\right) / b-2 \lambda a$, что отражает нарушение коллинеарности спинов системы вихрями при $T<T_{c}$. Соответственно, критическая температуpa для калибровочного поля смещается из $T_{c}$ в $T_{g}=T_{c}-2 \lambda b a / g^{2} \alpha$, и

$$
M_{e f f}^{2} \approx M^{2}=g^{2} \alpha\left(T_{g}-T\right) / b .
$$




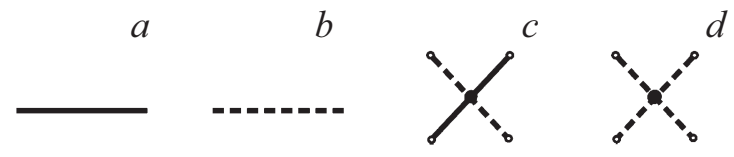

Рис. 1. $a)$ функция Грина векторного поля $\mathbf{A}, b)$ функцией Грина параметра порядка $\delta \Psi, c)$ схематическое представление вершины $g^{2}$, a $d$ ) графическое представление вершины $b$.

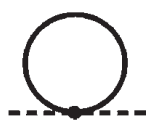

Рис. 2. Однопетлевой вклад в перенормировку массы поля высоких полей.

В температурном интервале $T_{c}>T>T_{g}$ система попадает в состояние, наделенное локальным упорядочением, но дальний порядок по-прежнему разрушается подвижными вихрями. Это состояние представляет собой вихревой газ и называется неупорядоченной фазой.

Если вмороженный беспорядок слабый и число вихрей невелико, то фазовый переход второго рода все же происходит, но при температуре несколько ниже, чем $T_{c}$. Другими словами, влияние индуцирующего вихри беспорядка приводит к перенормировке температуры фазового перехода, смещая ее вниз, $T_{c} \rightarrow T_{c}^{R}$.

Определим перенормированную температуру фазового перехода. Для этого перепишем гамильтониан системы (3) с учетом вихрей и малых флуктуаций, $\delta \Psi$, поля $\Psi, \Psi=\psi+\delta \Psi$ :

$$
\begin{aligned}
\mathscr{H}= & \frac{1}{2} \mathbf{A} \nabla^{2} \mathbf{A}-\frac{1}{2} M_{\text {eff }}^{2} \mathbf{A}^{2}+\frac{1}{2}|\nabla \delta \Psi|^{2} \\
& -\frac{1}{2} g^{2} \mathbf{A}^{2}|\delta \Psi|^{2}-\frac{1}{2} m^{2}|\delta \Psi|^{2}+\frac{b}{4}|\delta \Psi|^{4} .
\end{aligned}
$$

Функции Грина, входящие в этот гамильтониан полей, имеют следующий вид:

$$
\begin{gathered}
\langle\mathbf{A}(\mathbf{p}) \mathbf{A}(-\mathbf{p})\rangle_{A}=\frac{-\beta^{-1}}{\mathbf{p}^{2}+M_{0}^{2}}, \\
\langle\delta \Psi(\mathbf{p}) \delta \Psi(-\mathbf{p})\rangle=\frac{\beta^{-1}}{\mathbf{p}^{2}-m^{2}} .
\end{gathered}
$$

Эти функции Грина и вершины модели могут быть графически представлены в виде, показанном на рис. 1.

Определим перенормированную температуру фазового перехода. Диаграммное представление однопетлевого вклада в эту перенормировку дано на рис. 2. Используя свойства $d$-мерного интегрирования [31]

$$
\begin{aligned}
\int d^{d} \mathbf{p} \frac{\left(\mathbf{p}^{2}\right)^{\alpha}}{\left(\mathbf{p}^{2}+M^{2}\right)^{\gamma}} & =\pi^{d / 2} M^{d+2 \alpha-2 \gamma} \\
& \times \frac{\Gamma(\alpha+d / 2) \Gamma(\gamma-\alpha-d / 2)}{\Gamma(d / 2) \Gamma(\gamma)},
\end{aligned}
$$

можно показать, что петля на рис. 2 дает следующий вклад:

$$
\approx g^{2} \beta \int \frac{d^{3} \mathbf{p}}{(2 \pi)^{3}} \frac{\beta^{-1}}{\mathbf{p}^{2}+M^{2}(T)}=-g^{2}|M(T)|,
$$

поэтому

$$
\begin{aligned}
\alpha\left(T-T_{c}^{R}\right) & \approx \alpha\left(T-T_{c}\right)+\beta^{-1} g^{2}\left|M\left(T_{c}\right)\right| \\
& =\alpha\left(T-T_{c}\right)+\beta^{-1} g^{2} \sqrt{g^{2} \alpha\left(T_{c}-T_{g}\right) / b} .
\end{aligned}
$$

Поскольку $\quad M^{2}\left(T_{g}\right)=g^{2} \alpha\left(T_{g}-T_{c}\right) / b+2 \lambda a^{d-2}=0, \quad$ то перенормированная температура фазового перехода зависит от концентрации вихрей, $\lambda$ :

$$
T_{c}^{R}(\lambda)=T_{c}-\frac{g^{2}}{\beta \alpha} \sqrt{2 a \lambda} .
$$

Если $T_{c}^{R}>T_{g}$, система испытывает фазовый переход второго рода, при $T=T_{c}^{R}$. В противном случае, $T_{c}^{R}<T_{g}$, при $T=T_{g}$ система претерпевает фазовый переход, особенностью которого является то, что он является топологическим фазовым переходом, аналогичным переходу БКТ в двумерных системах [32], в котором параметр порядка не возникает при $T_{g}$, но радиус корреляции калибровочного поля расходится, при $T<T_{g}$ поле становится массивным и, как результат, система „замерзает“ в неупорядоченном состоянии, которое можно определить как стекольное состояние.

4.2. Анализ критического поведения в близи $T_{g}$. Для проверки нашего предположения о том, что при $T \gtrsim T_{g}$ происходит стекольный переход, исследуем поведение системы вблизи этой точки. В этом случае не существует стандартного параметра порядка Ландау, поэтому для описания критического поведения системы надо рассмотреть корреляционную функцию вихрей. При $\beta \ll 1$ и $p \rightarrow 0$ в импульсном представлении парная корреляционная функция вихрей может быть представлена следующим образом:

$$
\langle\mathbf{J}(\mathbf{p}) \mathbf{J}(-\mathbf{p})\rangle=\left\langle\langle\mathbf{J}(\mathbf{p}) \mathbf{J}(-\mathbf{p})\rangle_{J}\right\rangle_{A} \propto \exp \left[-\beta \frac{a^{d-4} J^{2} e^{i \mathbf{p r}}}{\mathbf{p}^{2}+M_{e f f}^{2}}\right] .
$$

Мы полагаем, что в неупорядоченной фазе, где $M_{e f f}^{2}<0$, вихревая корреляционная функция экспоненциально убывает с расстоянием $\mathbf{r},\langle\mathbf{J}(0) \mathbf{J}(\mathbf{r})\rangle \propto$ $\propto \exp \left(-|\mathbf{r}| / r_{c}\right)$, где корреляционный радиус, $r_{c}$, соответствует характерному размеру пары вихрь-антивихрь. В трехмерном случае $r_{c}$ это размер вихревых петель. Тогда можно видеть, что при $p \rightarrow 0$ корреляционный радиус может быть оценен в импульсном представлении как

$$
r_{c} \propto\left\langle\mathbf{J}_{1}(\mathbf{p}) \mathbf{J}_{2}(-\mathbf{p})\right\rangle_{p \rightarrow 0} \propto \exp \left[-\frac{a^{-2}}{M_{e f f}^{2}}\right] .
$$


Чтобы найти соответствующий радиус корреляции в 3D системе, мы используем уравнения (19) и (27) и получаем

$$
r_{c} \propto \exp \left[\frac{b}{\alpha(a g)^{2}} \frac{1}{T-T_{g}}\right]=\exp \left[\frac{1}{2 \lambda a^{3}} \frac{T_{c}-T_{g}}{T-T_{g}}\right] .
$$

Из полученного выражения видно, что расходимость корреляционного радиуса вихрей при $T \rightarrow T_{g}$ описывается соотношением Фогеля-Фулчера-Таммана, характерным для стекольного перехода. Заметим, что обычно критическое поведение стекол описывается в терминах времени релаксации. Здесь же расходится корреляционный радиус. В этом нет ничего удивительного, поскольку, как известно из критической динамики, рост корреляционной длины сопровождается соответствующей расходимостью времени релаксации, $\tau \sim r_{c}{ }^{z}$, где $z$ - динамический показатель $(z \approx 2)$ [33,34], что подразумевает критическое замедление системы вблизи $T_{g}$. Напомним, что дальнодействующая корреляция вихрей не означает какого-либо дальнего порядка самих спинов, $\left.\langle\Psi \Psi\rangle\right|_{r \rightarrow \infty} \neq 0$, поэтому никакого противоречия с существующими теоретическими представлениями о спиновых стеклах здесь нет.

Поскольку в данной работе мы ограничивамся статической теорией, мы не можем судить об эргодичности системы ниже $T_{g}$, чтобы доказать, что она находится в состоянии стекла. Поэтому мы приведем другие аргументы для подтверждения нашего вывода. Прежде всего отметим, что из-за наличия устойчивых вихрей любой дальний порядок в низкотемпературной фазе разрушается, $\langle\Psi\rangle=0$. Спиновая корреляционная функция $\left.\langle\Psi \Psi\rangle\right|_{r \rightarrow \infty}=0$, а длина спиновой корреляции мала, $\sim m^{-1}=\sqrt{g / 2 \lambda b a^{d-1}}$. Как мы уже упоминали выше, используя дисперсионное соотношение $\omega \propto p^{z}$ для динамики релаксации, можно оценить время релаксации, описываемое зависимостью ФФТ: $\tau \propto r_{c}{ }^{z} \propto \exp \left[E_{0}^{\prime}\left(T-T_{g}\right)^{-1}\right]$. Отсюда можно предположить, что переход при $T=T_{g}$ принадлежит к тому же классу фазовых переходов, что и стекольный переход в упругих средах [27]. Это свойство является характерной чертой стекольного перехода.

Другим критерием стекольного перехода в спиновой системе является характерное поведение линейной и нелинейной восприимчивостей при температуре перехода [35]. Чтобы определить эти свойства, добавим к гамильтониану нашей системы 20 внешнее поле $\mathbf{h}$

$$
\begin{aligned}
\mathscr{H}= & \frac{1}{2} \mathbf{A} \nabla^{2} \mathbf{A}-\frac{1}{2} M_{e f f}^{2} \mathbf{A}^{2}+\frac{1}{2}|\nabla \delta \Psi|^{2}-\frac{1}{2} g^{2} \mathbf{A}^{2}|\delta \Psi|^{2} \\
& -\frac{1}{2} m^{2}|\delta \Psi|^{2}+\frac{b}{4}|\delta \Psi|^{4}-\mathbf{h} \delta \Psi
\end{aligned}
$$

Линейная восприимчивость системы может быть выражена следующим образом: $\chi_{L}=\partial\langle\delta \Psi\rangle /\left.\partial \mathbf{h}\right|_{h \rightarrow 0, p \rightarrow 0}=$ $=\left.\beta\left\langle\Psi^{2}\right\rangle\right|_{p \rightarrow 0}$. Вблизи $T_{g}$ это значение перенормируется.

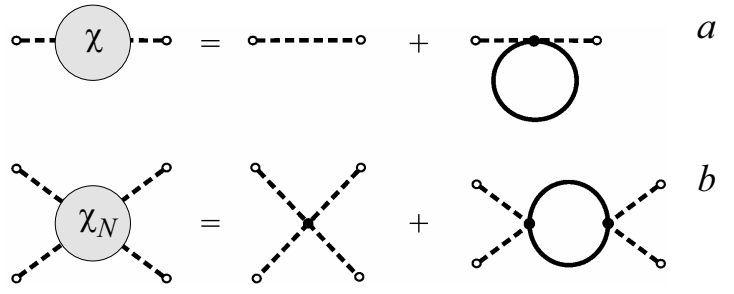

Pис. 3. a) Графическое представление однопетлевой аппроксимации линейной восприимчивости. Видно, что вблизи $T_{g}$ это значение в целом зависит от перенормировки $m^{2}$, что не приводит к появлению каких-либо расходимостей. $b$ ) Графическое представление однопетлевого приближения нелинейной восприимчивости.

В однопетлевом приближении оно может быть представлено в диаграммной форме, показанной на рис. 3, $a$. Петля поля Хигса дает $\approx \beta^{-1} g^{2}|M(T)|$, поэтому пропорциональный $M$ второй член в выражении

$$
\begin{aligned}
\chi_{L} & =\beta\left\langle\Psi^{2}\right\rangle_{p=0} \\
& =\beta\left\langle\delta \Psi^{2}\right\rangle_{p=0}^{0}+\beta|M| g^{2}\left[\left\langle\delta \Psi^{2}\right\rangle_{p=0}^{0}\right]^{2}+\ldots
\end{aligned}
$$

будет мал. В результате $\chi_{L} \approx \beta^{-1} m^{-2}$ $=\left[\alpha\left(T_{c}-T_{g}\right) / T_{g}\right]^{-1}$. Это значение конечно в $T_{g}$, что характерно для стекольного перехода, в отличие от бесконечно расходящегося значения при фазовом переходе второго рода.

Нелинейная восприимчивость может быть выражена следующим образом: $\chi_{N}=\partial^{3}\langle\Psi\rangle /\left.\partial \mathbf{h}^{3}\right|_{h \rightarrow 0, p \rightarrow 0}$ $=\beta^{3}\left\langle\Psi^{4}\right\rangle_{p=0}$. В однопетлевом приближении перенормировку этого значения можно диаграммно представить в виде, показанном на рис. $3, b$. Вблизи $T_{g}$ второй член дает расходящийся вклад, который доминирует. В результате нелинейная восприимчивость может быть оценена как $\chi_{N} \propto-\ln \left(T-T_{g}\right)$ при $T \rightarrow T_{g}^{+}$. Эта величина расходится в $T=T_{g}$, что также соответствует стекольному переходу в спиновых системах [35].

Сочетание конечной линейной восприимчивости с бесконечной нелинейной восприимчивостью в точке $T_{g}$ в отсутствие дальнего порядка, $\langle\Psi\rangle=0$, указывает на то, что $T_{g}$ является температурой стекольного перехода, см. [35-37,4,1,38,39]. Рассматриваемая физическая картина также согласуется с „теорией ограниченного роста доменов“ $[36,37]$ и с „калибровочной теорией стекла“ $[4,1]$. Кроме того, ранее, используя методы неравновесной критической динамики, мы показали, что фрустрированная трехмерная система, которая претерпевает фазовый переход второго рода или слабый фазовый переход первого рода, не достигает низкотемпературного упорядоченного состояния, но замерзает в неэргодическом состоянии стекла [39]. Принимая во внимание все изложенные аргументы, мы полагаем, что $T_{g}$ действительно является температурой стеклования. 


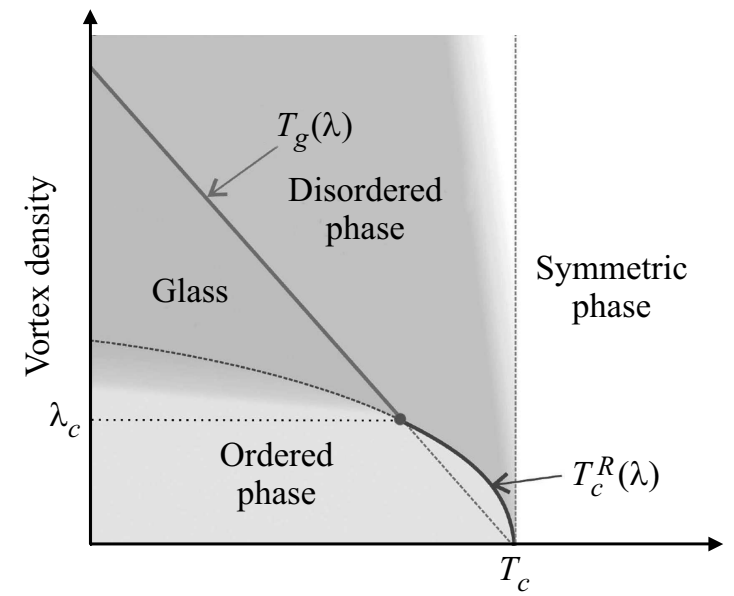

Pис. 4. Схематическая фазовая диаграмма трехмерной ХY-модели с вмороженным беспорядком. Линия $T_{c}^{R}(\lambda)$ обозначает перенормированную температуру фазового перехода второго рода, а линия $T_{g}(\lambda)-$ зависимость температуры стекольного перехода от $\lambda$. При $T_{c}^{R}>T_{g}$ трехмерная система подвергается фазовому переходу второго рода. При $T_{c}^{R}<T_{g}$ система переходит в стекольное состояние

\section{5. Заключение}

Мы построили калибровочную теорию трехмерной ХY-модели с вмороженным беспорядком, в которой было учтено формирование вихреподобных топологических возбуждений. Мы обнаружили, что при $T=T_{c}$ неупорядоченная ХY-модель в результате кроссовера приобретает ориентационную жесткость [34], что приводит к образованию вихрей. Мы расширили описание БКТ-подхода на трехмерную ХҮ-модель с вмороженным беспорядком, и продемонстрировали, что в ней происходит аналогичный по природе топологический фазовый переход. При $T<T_{c}$ в спиновой системе появляются вихри в виде вихревых линий, начинающихся и заканчивающихся на поверхностях системы, и вихревых петель произвольного размера [40]. В случае относительно сильного вмороженного беспорядка и, как результат, высокой плотности вихрей, превышающей некоторое критическое значение $\lambda_{c}$, дальнейшее охлаждение системы приводит к ее замерзанию в стекольном состоянии при $T_{g}(\lambda)>T_{c}^{R}(\lambda)$. Во время стеклования система демонстрирует критическое поведение Фогеля-Фулчера-Таммана. Однако, если концентрация вихрей не достаточно высока и остается ниже некоторого порогового значения $\lambda_{c}$, система претерпевает обычный фазовый переход второго рода. Качественный вид результирующей фазовой диаграммы показан на рис. 4.

В заключение следует отметить, что топологический фазовый переход, связанный с появлением топологически устойчивых линейных возмущений (вихревых линий) бесконечной длины, хорошо известен как модель плавления кристалла [41]. По сути, переход к стеклянному состоянию из высокотемпературной жидкой фазы также является топологическим фазовым переходом. Однако есть существенное различие между топологическим плавлением и стеклянным переходом. В первом случае особые термически стабильные возмущения возникают в результате теплового возбуждения, тогда как в спиновых стеклах они также существуют при низких температурах из-за закаленного беспорядка в состоянии с замороженной конфигурацией [5].

\section{Список литературы}

[1] N. Rivier. Rev. Brasileira Fis., 15, 4, 311 (1985).

[2] D. Chowdhury. Spin Glasses and Other Frustrated Systems. World Scientific. ISBN 9971-50-029-9.

[3] G. Toulouse. Commun. Phys. 2, 115 (1977).

[4] N. Rivier, D.M. Duffy. J. Phys. 43, 293 (1982).

[5] I.E. Dzyaloshinskii, G.E. Volovik. J. Phys. 39, 693 (1978).

[6] И.Е. Дзялошинский, С.П. Обухов. ЖЭТФ 83, 2, 813 (1982).

[7] J.A. Hertz. Phys. Rev. B 18, 4875 (1978).

[8] В.Л. Березинский. ЖЭТФ 61, 3, 1144 (1972).

[9] J.M. Kosterlitz, D.J. Thouless. J. Phys. C 6, 1181 (1973).

[10] J.M. Kosterlitz, D.J. Thouless. J. Phys. C 5, L124 (1972).

[11] J.M. Kosterlitz. J. Phys. C 7, 1046 (1974).

[12] J. José, L.P. Kadanoff, S. Kirkpatric, D.R. Nelson. Phys. Rev. B 16, 1217 (1977).

[13] P.W. Anderson. Lectures on amorphous systems. In: Les Houches, Session XXXI, 1978 - La matière mal condensée/III-condensed matter, 1978 / Ed. R. Balian, Gerard Toulouse, R. Maynard (North-Holland, Amsterdam, 1978).

[14] G. Kohring, R.E. Shrock, P. Wills. Phys. Rev. Lett. 57, 1358 (1986).

[15] P. Olsson. Phys. Rev. B 52, 4526 (1995).

[16] P. Olsson. Phys. Rev. Lett. 91, 077002-1 (2003).

[17] M. Camarda, F. Siringo, R. Pucci, A. Sudbo, J. Hove. Phys. Rev. B 74, 104507 (2006).

[18] D.A. Garanin, E.M. Chudnovsky, T. Proctor. Phys. Rev. B 88, 224418 (2013).

[19] N. Mermin, H. Wagner. Phys. Rev. Lett. 17, 1133 (1966).

[20] P.C. Hohenberg. Phys. Rev. 158, 383 (1967).

[21] A. Zee. Quantum Field Theory in a Nutshell. Princeton University Press, Princeton (2010). ISBN 9780691140346.

[22] Р.Л. Стратонович. ДАН СССР. 157, 6, 1097 (1957).

[23] J. Hubbard. Phys. Rev. Lett. 3, 77 (1959).

[24] P. Minnhagen. Rev. Mod. Phys. 59, 1001 (1987).

[25] J. Villain. J. Phys. C 11, 745 (1978).

[26] J. Kierfeld, V.M. Vinokur. Phys. Rev. B 61, R14928 (2000).

[27] D.R. Nelson. Phys. Rev. B 28, 5515 (1983).

[28] G. Parisi, N. Sourlas. Phys. Rev. Lett. 43, 744 (1979).

[29] А.М. Поляков. Калибровочные поля и струны. ИТФ им. Ландау, М. (1994).

[30] A.M. Tsvelik. Quantum Field Theory in Condensed Matter Physics. Cambridge University Press, Cambridge (1998). ISBN 0521589894.

[31] J.C. Collins. Renormalization, Cambridge University Press, Cambridge (1984) 448 p. ISBN 0521311772.

[32] M.G. Vasin, V.N. Ryzhov, V.M. Vinokur. arXiv:1712.00757. В печати. 
[33] P.C. Hohenberg, B.I. Halperin. Rev. Mod. Phys. 49, 3, 435 (1977).

[34] А.3. Паташинский, В.Л. Покровский. Флуктуационная теория фазовых переходов. Наука, М. (1982). 382 с.

[35] K. Binder, A.P. Young. Rev. Mod. Phys. 58, 4, 801 (1986).

[36] D. Kivelson, G. Tarjus. Phyl. Mag. B 77, 245 (1998).

[37] G. Tarjus, S.A. Kivelson, Z. Nussinov, P. Viot. J. Phys: Cond. Matter, 17, 50, R1143 (2005).

[38] Z. Nussinov. Phys. Rev. B 69, 014208 (2004).

[39] M.G. Vasin. J. Statistic. Mech.: Theory and Exp. P05009 (2011).

[40] С.П. Обухов. ЖЭТФ 83, 1978 (1982); [S.P. Obukhov. Sov. Phys. JETP 56, 5, 1144 (1982)].

[41] B.I. Halperin. Statistical Mechanics of Topological Defects. In: Les Houches. Session XXXV. Physics of Defects/ Ed

R. Balian, M. Kleman, J.-P. Poirier. North-Holland Publishing Company (1981). 